cally assessed for EBV infection for 3 years had little effect on the frequency of silent seroconversion but greatly reduced the frequency of IM (20). This is an important result because it suggests that a vaccine to prevent IM might actually work and also because it points the way to the most sensible vaccine strategy, namely to try to prevent IM but not the normal silent infection with EBV. One can easily envisage how a partially effective gp350 vaccine might prevent IM but not silent EBV infection - if a high viral dose proves to be required for the development of IM, the immune response induced by the vaccine might be able to neutralize most of this virus (preventing IM) but not completely protect against infection. There is therefore an increasing case for further efforts to develop an EBV vaccine that could be given to EBV-seronegative teenagers or adults to try to prevent the development of IM.

Address correspondence to: Paul J. Farrell, Department of Virology, Imperial College London, St Mary's Campus, Norfolk Place, London W2 1PG, United Kingdom. Phone: 44-020-7594-2005; Fax: 44-020-7594-3973; E-mail: p.farrell@imperial.ac.uk.
1. Rickinson, A.B., and Kieff, E. 2001. Epstein-Barr virus. In Fields virology. D. Knipe and P.M. Howley, editors. Lippincott Williams \& Wilkins. Philadelphia, Pennsylvania, USA. 2575-2627.

2. Thorley-Lawson, D.A., and Gross, A. 2004. Persistence of the Epstein-Barr virus and the origins of associated lymphomas. N. Engl. J. Med. 350:1328-1337.

3. Mancao, C., and Hammerschmidt, W. 2007. Epstein-Barr virus latent membrane protein $2 \mathrm{~A}$ is a B-cell receptor mimic and essential for B-cell survival. Blood. In press.

4. Laichalk, L.L., and Thorley-Lawson, D.A. 2005 Terminal differentiation into plasma cells initiates the replicative cycle of Epstein-Barr virus in vivo. J. Virol. 79:1296-1307.

5. Hislop, A.D., Taylor, G.S., Sauce, D., and Rickinson, A.B. 2007. Cellular responses to viral infection in humans: lessons from Epstein-Barr virus. Annu. Rev. Immunol. 25:587-617.

6. Sauce, D., et al. 2006. EBV-associated mononucleosis leads to long-term global deficit in T-cell responsiveness to IL-15. Blood. 108:11-18.

7. Hjalgrim, H., et al. 2003. Characteristics of Hodgkin's lymphoma after infectious mononucleosis. N. Engl. J. Med. 349:1324-1332.

8. Hjalgrim, H. 2007. Infectious mononucleosis, childhood social environment, and risk of Hodgkin lymphoma. Cancer Res. 67:2382-2388.

9. Jarrett, R.F., et al. 2005. Impact of tumor EpsteinBarr virus status on presenting features and outcome in age-defined subgroups of patients with classic Hodgkin lymphoma: a population-based study. Blood. 106:2444-2451.

10. Young, L.S., and Murray, P.G. 2003. EpsteinBarr virus and oncogenesis: from latent genes to tumours. Oncogene. 22:5108-5121.

11. McAulay, K.A., et al. 2007. HLA class I polymorphisms are associated with development of infec- tious mononucleosis upon primary EBV infection. J. Clin. Invest. 117:3042-3048. doi:10.1172/JCI32377.

12. Diepstra, A., et al. 2005. Association with HLA class I in Epstein-Barr-virus-positive and with HLA class III in Epstein-Barr-virus-negative Hodgkin's lymphoma. Lancet. 365:2216-2224.

13. Niens, M., et al. 2007. HLA-A*02 is associated with a reduced risk and HLA-A*01 with an increased risk of developing EBV-positive Hodgkin lymphoma. Blood. In press.

14. Helminen, M., Lahdenpohja, N., and Hurme, M. 1999. Polymorphism of the interleukin-10 gene is associated with susceptibility to Epstein-Barr virus infection. J. Infect. Dis. 180:496-499.

15. Wu, M.S., et al. 2002. Tumor necrosis factor-alpha and interleukin-10 promoter polymorphisms in Epstein-Barr virus-associated gastric carcinoma. J. Infect. Dis. 185:106-109.

16. Hurme, M., and Helminen, M. 1998. Polymorphism of the IL-1 gene complex in Epstein-Barr virus seronegative and seropositive adult blood donors. Scand. J. Immunol. 48:219-222.

17. Brauninger, A., et al. 2006. Molecular biology of Hodgkin's and Reed/Sternberg cells in Hodgkin's lymphoma. Int. J. Cancer. 118:1853-1861.

18. Crawford, D.H., et al. 2006. A cohort study among university students: identification of risk factors for Epstein-Barr virus seroconversion and infectious mononucleosis. Clin. Infect. Dis. 43:276-282.

19. Tierney, R.J., et al. 2006. Multiple Epstein-Barr virus strains in patients with infectious mononucleosis: comparison of ex vivo samples with in vitro isolates by use of heteroduplex tracking assays. J. Infect. Dis. 193:287-297.

20. Moutschen, M., et al. 2007. Phase I/II studies to evaluate safety and immunogenicity of a recombinant gp350 Epstein-Barr virus vaccine in healthy adults. Vaccine. 25:4697-4705.

\title{
Overstaying their welcome: defective CX3CR1 microglia eyed in macular degeneration
}

\author{
Jing Chen, Kip M. Connor, and Lois E.H. Smith
}

Department of Ophthalmology, Children's Hospital Boston, Harvard Medical School, Boston, Massachusetts, USA.

\begin{abstract}
Age-related macular degeneration (AMD), the most common cause of blindness in the elderly, is characterized by degeneration of the macula and can lead to loss of fine color vision. Alterations in inflammatory and immune system pathways, which arise from genetic differences, predispose individuals to AMD. Yet the mechanism of disease progression with respect to inflammation is not fully understood. In this issue of the JCI, the study by Combadière and colleagues shows that $\mathrm{CX} 3 \mathrm{C}$ chemokine receptor 1-deficient (CX3CR1-deficient) mice have abnormal microglia that accumulate beneath the retina and contribute to the progression of AMD (see the related article beginning on page 2920).
\end{abstract}

Nonstandard abbreviations used: AMD, age-related macular degeneration; $\mathrm{Ccl} 2, \mathrm{CC}$ chemokine ligand 2; Ccr2, CC chemokine receptor 2; CX3CL1, CX3C chemokine ligand 1; CX3CR1, CX3C chemokine receptor 1; RPE, retinal pigment epithelium.

Conflict of interest: The authors have declared that no conflict of interest exists.

Citation for this article: J. Clin. Invest. 117:2758-2762 (2007). doi:10.1172/JCI33513.
Age-related macular degeneration (AMD), a degenerative disease of the retina, is the most common cause of visual impairment in the elderly in the developed world (1). The prevalence of AMD is expected to increase as the population ages. AMD is characterized by degeneration of the macula, an area in the central retina with the highest concen- tration of cones that is responsible for highacuity and color vision (Figure 1). Damage to the macula can cause profound loss of fine vision (Figure 1A). Genetic factors identified for AMD risk involve regulation of the inflammatory, complement, and chemokine pathways, including the fractalkine receptor CX3C chemokine receptor 1 (CX3CR1) (2). CX3CR1 is expressed in microglia, the resident macrophages in the CNS, and the retina $(3,4)$ and mediates migration and adhesion of these cells in response to CX3C chemokine ligand 1 (CX3CL1) (5).

In this issue of the JCI, a new study by Combadière et al. (6) confirmed that in humans, the CX3CR1 M280 allele increases the risk of human AMD and that microglia isolated from these individuals migrate defectively. The authors also showed that in 

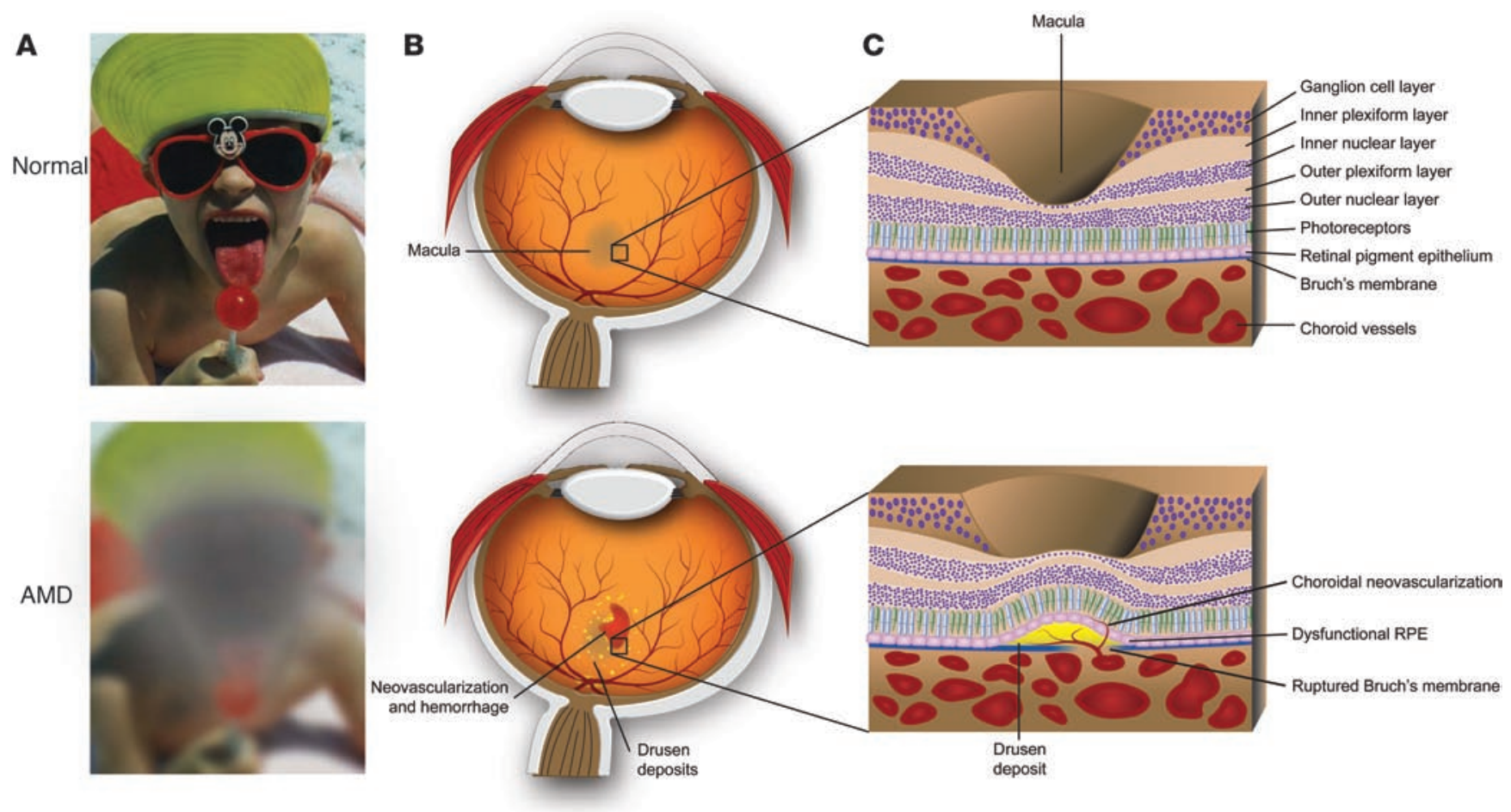

\section{Figure 1}

Loss of central vision in AMD patients. (A) Representation of the visual field seen by a person with normal vision and by an AMD patient with a loss of central color vision. (B) Schematic of a normal human eye compared with an AMD eye depicting deposits of drusen (yellow spots) and choroidal neovascularization with subretinal hemorrhage in the macula (red). (C) Schematic cross-section of a normal eye through the macula shows retinal neuronal layers, RPE, Bruch's membrane, and choroid vessels. In AMD, the intimate relation among photoreceptors, RPE, and choroid is disrupted by drusen (lipid and cellular debris-containing deposits). The formation of drusen separates RPE from Bruch's membrane and the underlying choroidal vessels in association with RPE atrophy and photoreceptor degeneration (hallmarks of dry AMD). In wet AMD with choroidal neovascularization, abnormal leaky choroidal vessels proliferate and penetrate the altered Bruch's membrane protruding into the subretinal space, causing hemorrhage and rapid loss of vision.

CX3CR1-deficient mice, microglia accumulate in the subretinal space, evoking morphological and pathological features similar to those observed in human AMD. The data suggest an important role of CX3CR1 and of microglia in the pathogenesis of AMD.

\section{The retina functions as film in a camera}

The retina is composed of several transparent neuronal layers. Light focused through the cornea and lens passes across these layers to interact with the pigments in the photoreceptor outer segments, causing a chemical transformation. This ultimately propagates a series of action potentials across the retinal and brain neurons, and we perceive an image. The photoreceptors are dependent upon a well-functioning adjoining retinal pigment epithelium (RPE), which phagocytoses the spent photoreceptor outer segments that are shed daily to recycle photopigments (such as rhodopsin) and membrane lipids. The
RPE also transports nutrients and breakdown products between the photoreceptors and the adjoining choroidal vascular plexus, separated from the RPE by Bruch's membrane (Figures 1 and 2). Therefore the photoreceptors, choroidal vessels, and RPE have an interdependent relationship, and loss of any one component causes dysfunction of the others. This dysfunction is often seen first in the macula because the densely packed photoreceptors here have the highest oxygen consumption and metabolic rate of any tissue in the body (7).

\section{In AMD the intimate relationship of RPE, photoreceptors, and choroid is interrupted}

Clinically, AMD begins with the asymptomatic appearance of drusen, seen as white or yellow spots beneath the retina. What triggers the formation of drusen is not completely understood, and not all drusen formation leads to AMD. Drusen are deposits of lipid and cellular debris that are found beneath the RPE on Bruch's membrane (Figures 1 and 2). These deposits increasingly separate the RPE from the underlying choroidal vascular bed, interrupting the function of the RPE and leading to photoreceptor degeneration, a hallmark of dry AMD (Figures 1 and 2). In wet AMD, its most devastating form, abnormal leaky blood vessels grow from the choroidal vasculature through the disrupted Bruch's membrane and RPE into the subretinal space beneath the photoreceptors, causing profound visual loss (Figures 1 and 2).

\section{Role of inflammatory cells in AMD}

Many risk factors for AMD are related to inflammation. These include environmental factors, such as smoking and low omega-3 fatty acid intake $(8,9)$, and genetic factors, such as polymorphisms complement factor $\mathrm{H}$ (10) and CX3CR1 (2), the chemokine CX3CL1 receptor. Chemokines are small proteins that induce directed chemotaxis in nearby responsive immune 


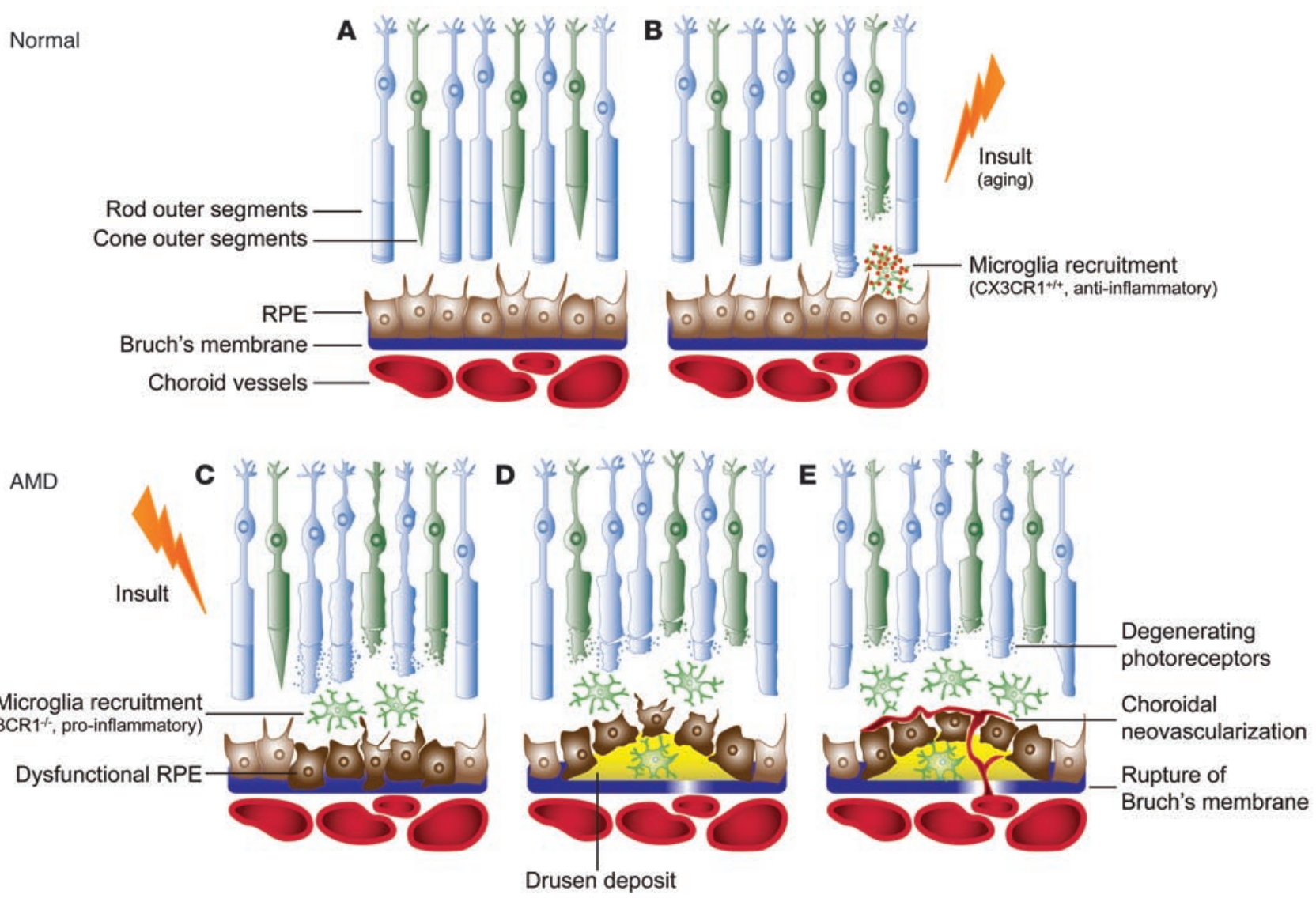

Figure 2

Proposed role of CX3CR1 and microglia in AMD. (A) In the normal retina, rod and cone photoreceptor outer segments are composed of thousands of photopigment-containing membrane discs that are continuously shed from the tips of the cells and recycled. The discarded discs are removed by RPE phagocytosis and reprocessed. (B) Additionally, microglia expressing CX3CR1 phagocytize cellular debris caused by daily insults (such as aging and cellular stress) and help maintain a healthy eye. (C) AMD resulting from loss of CX3CR1 expression is initiated by the same daily insults. However, as reported by Combadière et al. (6), when microglia lacking CX3CR1 are recruited to the area of damage they remain there, in part because of abnormal migration. CX3CR1-deficient microglia are proinflammatory (versus antiinflammatory CX3CR1-replete microglia; ref. 16) and recruit other proinflammatory cells. This response exacerbates the cellular damage that occurs with normal degeneration. (D) The increased accumulation of proinflammatory, CX3CR1-negative (16) microglia in the subretinal space appears to add to the formation of drusen (6), which contribute to loss of RPE function and photoreceptor degeneration (dry AMD). (E) Finally, as drusen build up and separate the RPE from the choroidal vasculature, the resulting hypoxia triggers proangiogenic signals, which foster choroidal neovascularization (wet AMD).

cells. Macrophage chemoattractants as well as elevated levels of inflammatory mediators and activated complement components are found in drusen samples from AMD patients $(11,12)$.

However, the mechanism of AMD with respect to inflammation and chemoattractants is not well defined. In their current study, Combadière and coworkers (6) expand our understanding of the role of chemokines in AMD. They suggest that defective microglia with abnormal CX3CR1 function contribute to the formation of drusen in AMD and subsequently promote photoreceptor loss and choroidal neovascularization. The authors show that, compared with mice with normal microglia, mice deficient in CX3CR1 had subretinal accumulation of microglia and drusenlike deposits after insults to the retina including senescence, light-induced retinal degeneration, and laser damage to rupture Bruch's membrane. The drusen-like material contained photoreceptor pigment (rhodopsin), indicating contribution from shed rod photoreceptor outer segments, as well as markers indicating contribution from accumulated defective microglia lacking CX3CR1 expression. Because these deposits precede photoreceptor degeneration and choroidal neovascularization (Figure 2), the authors suggest that these drusen could cause these AMD features. This study provides new insight into the putative role of microglia in drusen formation and into the role of inflammation in the pathogenesis of AMD, but raises many questions as well. How and why do these defective microglia accumulate - through decreased egress alone or increased attraction to the area? How do CX3CR1-defective microglia cause photoreceptor degeneration and choroidal neovascularization? How do these results reconcile with studies showing that decreased macrophage recruitment increases aspects of AMD?

\section{Are microglia the cause or the cure for AMD?}

In aged transgenic mice lacking CC chemokine ligand $2(\mathrm{Ccl} 2)$ or its cognate receptor 
CC chemokine receptor 2 (Ccr2), both molecules involved in chemoattraction of macrophages and/or microglia, some features of AMD (drusen-like deposits and choroidal neovascularization) have been reported, suggesting that macrophage recruitment by $\mathrm{Ccl} 2$ or $\mathrm{Ccr} 2$ confers protection against the formation of AMD (13). Mice deficient in IL-10 - a potent inhibitor of cytokine and chemokine production - with inhibited recruitment of macrophages in retina have increased choroidal neovascularization (14). These results suggest that macrophage recruitment into the subretinal space is necessary to remove extracellular deposits and prevent the formation of drusen. Yet global depletion of macrophages and microglia in mice reduces laser-induced choroidal neovascularization (15). In contrast, the results reported here by Combadière and coworkers (6) suggest that accumulated CX3CR1deficient microglia secondary to increased recruitment or impaired egress from the retina can form drusen and cause photoreceptor degeneration and choroidal neovascularization, all aspects of AMD.

CX3CR1 is involved in leukocyte recruitment, and previous studies have shown that its loss leads to inhibition of leukocyte migration (5). It is likely that an alternate chemoattractant molecule, like that of $\mathrm{Ccr} 2$, is involved in recruitment of these cells into areas of retinal damage. But how does accumulation of CX3CR1-deficient microglia cause AMD-like symptoms?

Perhaps it is because macrophages can have many different and even opposite functions depending on their activation state. Given this possibility, the results of the present study fit with the recent finding in muscle that decreased expression of CX3CR1 results in macrophages with a proinflammatory phenotype compared with macrophages with high levels of CX3CR1, which have an antiinflammatory phenotype (16). It is reasonable to speculate that the accumulation in the subretinal space of resident microglia with reduced CX3CR1 function might therefore contribute to lowgrade inflammation, leading to the recruitment of other inflammatory cells including proangiogenic, bone marrow-derived macrophages, which in turn induce choroidal neovascularization and the pathology of AMD. Prolonged presence of these cells in the subretinal space may also spur phagocytosis of lipids normally processed by the RPE. These bloated microglia trapped subretinally might become dysfunctional and degenerate, contributing to the accu- mulation of extracellular cellular debris and to the early sign of drusen formation. Although the present study (6) raises interesting possibilities regarding the mechanism of drusen formation, the exact contribution of microglia and circulating macrophages to retinal degeneration and choroid neovascularization remains undefined.

\section{Current treatment of AMD}

The study by Combadière and coworkers (6) is an important contribution to the current inflammatory theory in AMD. A clear understanding of the cellular and molecular mechanisms of AMD could bring a major shift in our approach to disease treatment and prevention. Currently the only treatment to slow the progression of dry AMD to wet AMD is dietary supplementation with vitamins and antioxidants (17), which is consistent with the role of oxidation stress in this pathological process (18). Recently, higher intake of dietary lipids thought to be antiinflammatory (omega-3 long-chain polyunsaturated fatty acids and fish) (9) have been found to be associated with decreased likelihood of developing wet AMD (8). For wet AMD, the treatments focus on suppression of choroidal neovascularization, with laser ablation, photodynamic therapy, or, increasingly, anti-VEGF angiogenesis inhibitors. However, these treatments do not address the underlying cause of AMD, and preventive and nondestructive therapies are much more desirable.

A twin study by Seddon et al. found that genetic factors explain $46 \%-71 \%$ of the variation in AMD disease severity, while environment explains 19\%-42\% (19), so manipulation of some pathways involved in genetic risk could have significant benefit. But the possibility of manipulating the CX3CR1/CX3CL1 pathway specifically to prevent the development of AMD is uncertain at the moment. CX3CL1-deficient mice are protected against development of atherosclerosis (20), and CX3CL1 mediates antitumor responses (21), so manipulation of the CX3CR1/CX3CL1 pathway in AMD is complex and might disrupt the physiological process of microglia and/or macrophage recruitment and clearing of subretinal debris. Nevertheless, the study by Combadière and coworkers (6) suggests a new direction. Further understanding of the molecular basis of the pathogenesis of AMD might lead to new treatment based on inhibition of complement factors that promote choroidal neovascularization as well as pharmacological targeting of chemoattractant cytokines.

\section{Acknowledgments}

We thank Christopher M. Aderman for excellent technical help and for preparing figures for this manuscript. The authors' research is generously supported by the $\mathrm{V}$. Kann Rasmussen Foundation as well as by NIH grants EY008670, EY017017, and EY14811 (L.E.H. Smith); by NIH grants 1F32 EY017789 and 5 T32 EY07145 (K.M. Connor); and by Children's Hospital Boston Mental Retardation and Developmental Disabilities Research Center (NIH grant P01 HD18655). We thank the Juvenile Diabetes Research Foundation for fellowship support (J. Chen). This work was also supported by the Research to Prevent Blindness Lew Wasserman Merit Award (L.E.H. Smith).

Address correspondence to: Lois E.H. Smith, Department of Ophthalmology, Fegan 4, Children's Hospital, 300 Longwood Avenue, Boston, Massachusetts 02115, USA. Phone: (617) 919-2529; Fax: (617) 730-0392; E-mail: lois.smith@ childrens.harvard.edu.

1. Smith, W., et al. 2001. Risk factors for age-related macular degeneration: pooled findings from three continents. Ophthalmology. 108:697-704.

2. Tuo, J., et al. 2004. The involvement of sequence variation and expression of CX3CR1 in the pathogenesis of age-related macular degeneration. FASEB J. 18:1297-1299.

3. Cardona, A.E., et al. 2006. Control of microglial neurotoxicity by the fractalkine receptor. Nat. Neurosci. 9:917-924.

4. Checchin, D., Sennlaub, F., Levavasseur, E., Leduc, M., and Chemtob, S. 2006. Potential role of microglia in retinal blood vessel formation. Invest. Ophthalmol. Vis. Sci. 47:3595-3602.

5. Imai, T., et al. 1997. Identification and molecular characterization of fractalkine receptor CX3CR1, which mediates both leukocyte migration and adhesion. Cell. 91:521-530.

6. Combadière, C., et al. 2007. CX3CR1-dependent subretinal microglia cell accumulation is associated with cardinal features of age-related macular degeneration. J. Clin. Invest. 117:2920-2928. doi:10.1172/JCI31692.

7. Walls, G.L. 1963. The vertebrate eye and its adaptive radiation. Hafner Publishing Company. New York, New York, USA. 652.

8. SanGiovanni, J.P., et al. 2007. The relationship of dietary lipid intake and age-related macular degeneration in a case-control study: AREDS Report No. 20. Arch. Ophthalmol. 125:671-679.

9. Connor, K.M., et al. 2007. Increased dietary intake of omega-3-polyunsaturated fatty acids reduces pathological retinal angiogenesis. Nat. Med. 13:868-873.

10. Klein, R.J., et al. 2005. Complement factor $\mathrm{H}$ polymorphism in age-related macular degeneration. Science. 308:385-389.

11. Anderson, D.H., Mullins, R.F., Hageman, G.S., and Johnson, L.V. 2002. A role for local inflammation in the formation of drusen in the aging eye. Am.J. Ophthalmol. 134:411-431. 
12. Mullins, R.F., Russell, S.R., Anderson, D.H., and Hageman, G.S. 2000. Drusen associated with aging and age-related macular degeneration contain proteins common to extracellular deposits associated with atherosclerosis, elastosis, amyloidosis, and dense deposit disease. FASEB J. 14:835-846.

13. Ambati, J., et al. 2003. An animal model of agerelated macular degeneration in senescent Ccl-2- or Ccr-2-deficient mice. Nat. Med. 9:1390-1397.

14. Apte, R.S., Richter, J., Herndon, J., and Ferguson, T.A. 2006. Macrophages inhibit neovascularization in a murine model of age-related macular degeneration. PLoS Med. 3:e310.

15. Espinosa-Heidmann, D.G., et al. 2003. Macrophage depletion diminishes lesion size and severity in experimental choroidal neovascularization. Invest. Ophthalmol. Vis. Sci. 44:3586-3592.

16. Arnold, L., et al. 2007. Inflammatory monocytes recruited after skeletal muscle injury switch into antiinflammatory macrophages to support myogenesis. J. Exp. Med. 204:1057-1069.

17. 2001. A randomized, placebo-controlled, clinical trial of high-dose supplementation with vitamins $\mathrm{C}$ and $\mathrm{E}$, beta carotene, and zinc for age-related macular degeneration and vision loss: AREDS report no. 8. Arch. Ophthalmol. 119:1417-1436.

18. Beatty, S., Koh, H., Phil, M., Henson, D., and Boulton, M. 2000. The role of oxidative stress in the pathogenesis of age-related macular degeneration. Surv. Ophthalmol. 45:115-134.

19. Seddon, J.M., Cote, J., Page, W.F., Aggen, S.H., and Neale, M.C. 2005. The US twin study of age-related macular degeneration: relative roles of genetic and environmental influences. Arch. Ophthalmol. 123:321-327.

20. Teupser, D., et al. 2004. Major reduction of atherosclerosis in fractalkine (CX3CL1)-deficient mice is at the brachiocephalic artery, not the aortic root. Proc. Natl. Acad. Sci. U. S. A. 101:17795-17800.

21. Lavergne, E., et al. 2003. Fractalkine mediates natural killer-dependent antitumor responses in vivo. Cancer Res. 63:7468-7474

\title{
Why targeted therapy hasn't worked in advanced cancer
}

\author{
Jack L. Arbiser \\ Department of Dermatology and Winship Cancer Institute, Emory University School of Medicine, and Atlanta Veterans Administration Medical Center, \\ Atlanta, Georgia, USA.
}

\begin{abstract}
In this issue of the JCI, Nissen et al. report that a reciprocal interaction exists between the growth factors FGF2 and PDGF-BB, causing tumors to exhibit increased angiogenesis and metastatic potential (see the related article beginning on page 2766). Both FGF2 and PDGF-BB signal through tyrosine kinase receptors, which have been the target of tyrosine kinase inhibitors for cancer therapies. These inhibitors are usually small molecules that inhibit the kinase activity of a receptor or nonreceptor tyrosine kinase, preventing downstream signaling. The results of this study shed light on why tyrosine kinase inhibitors have been useful for the treatment of only a small number of advanced cancers. Currently, a major focus of pharmaceutical companies is to develop ever more potent and specific tyrosine kinases. The results presented here suggest that this approach may not be successful.
\end{abstract}

Tyrosine kinases are a large family of enzymes that phosphorylate target proteins, resulting in either activation or inactivation of these proteins. This family includes many peptide receptors as well as nonreceptor proteins and is well represented in oncogenic fusion proteins, such as the BCR-ABL protein, which is generated from the Philadelphia chromosome. These proteins activate multiple signaling pathways, including those involving PI3K, phospholipase $\mathrm{C} \gamma$, MAPK, and STAT activation and the generation of reactive oxygen species. The study by Nissen et al. in this issue of the JCI demonstrates that activation of a combination of several tyrosine kinase receptors results in a high-

Nonstandard abbreviations used: Ang-2, angiopoietin 2; CML, chronic myelogenous leukemia; FGFR1, FGF receptor 1 .

Conflict of interest: The author has declared that no conflict of interest exists.

Citation for this article: J. Clin. Invest. 117:2762-2765 (2007). doi:10.1172/JCI33190. ly angiogenic and metastatic phenotype and that the functions of these tyrosine kinase receptors is nonredundant in vivo (1). These findings may help explain why tyrosine kinases have been successful primarily in early malignancies but less so in advanced cancers.

In the first decade of the twenty-first century, the focus in the field of oncology has been on developing of targeted therapy against tyrosine kinases. This began with the use of the tyrosine kinase inhibitor imatinib, which has shown impressive activity against chronic myelogenous leukemia (CML), and has subsequently been successful in the treatment of other malignancies including gastrointestinal stromal tumors and mastocytosis (2). Other specific tyrosine kinase inhibitors have since appeared, including sorafenib, which was designed to inhibit the mutant $B$-raf oncogene in melanoma (3), in addition to both small molecule and antibody-mediated inhibitors of EGFR (4). EGFR, a member of the tyrosine kinase receptor family, is expressed in a wide variety of epithelial tumors in humans, including breast cancer and non-small cell carcinoma of the lung, calling attention to it as a druggable target. More recently, clinical results have identified some of the shortcomings of such targeted therapies. While imatinib is highly effective against early CML, it is less effective against blast crisis CML (2). Sorafenib was found to be relatively ineffective against melanoma in clinical trials (3), and EGFR tyrosine kinase inhibitors have been found to be most efficacious only in a small subset of patients - often female Japanese nonsmokers with bronchoalveolar histology $(4,5)$. While one response to these failures has been sustained efforts to identify more potent inhibitors, the study by Nissen et al. (1) should give drug developers pause. These authors show that it is the nonredundancy of tyrosine kinases such as FGF receptor 1 (FGFR1) and PDGFR, rather than the potency of the tyrosine kinase inhibitor, that is the main enemy of targeted therapy in cancer. Yet there is room for optimism if one uses combination or sequential tyrosine kinase inhibitor therapy instead of targeted monotherapy. Thus a "dirtier" approach to tyrosine kinase therapy is suggested, referring to the use of both promiscuous tyrosine kinase inhibitors, which inhibit more than one tyrosine kinase as well as a combination of tyrosine kinase inhibitors, and other signaling inhibitors, such as rapamycin. 